\title{
Skeletal muscle-specific overexpression of SIRT1 does not enhance whole-body energy expenditure or insulin sensitivity in young mice
}

\author{
A. T. White • C. E. McCurdy • A. Philp • D. L. Hamilton • \\ C. D. Johnson • S. Schenk
}

Received: 13 December 2012 /Accepted: 22 March 2013 / Published online: 19 April 2013

(C) Springer-Verlag Berlin Heidelberg 2013

\begin{abstract}
Aims/hypothesis The $\mathrm{NAD}^{+}$-dependent protein deacetylase sirtuin (SIRT)1 is thought to be a key regulator of skeletal muscle metabolism. However, its precise role in the regulation of insulin sensitivity is unclear. Accordingly, we sought to determine the effect of skeletal muscle-specific overexpression of SIRT1 on skeletal muscle insulin sensitivity and whole-body energy metabolism.

Methods At 10 weeks of age, mice with muscle-specific overexpression of SIRT1 and their wild-type littermates were fed a standard diet with free access to chow or an energy-restricted ( $60 \%$ of standard) diet for 20 days. Energy expenditure and body composition were measured by indirect calorimetry and magnetic resonance imaging, respectively. Skeletal muscle insulin-stimulated glucose uptake was measured ex vivo in soleus and extensor digitorum
\end{abstract}

A. T. White $\cdot$ C. D. Johnson $\cdot$ S. Schenk $(\bowtie)$

Department of Orthopaedic Surgery, School of Medicine,

University of California San Diego, 9500 Gilman Drive MC0863,

La Jolla, CA 92093-0863, USA

e-mail: sschenk@ucsd.edu

\section{A. T. White}

Biomedical Sciences Graduate Program,

University of California San Diego, La Jolla, CA, USA

C. E. McCurdy

Department of Pediatrics, University of Colorado Denver,

School of Medicine, Aurora, CO, USA

A. Philp

School of Sport and Exercise Sciences,

College of Life and Environmental Sciences,

University of Birmingham, Birmingham, UK

D. L. Hamilton

Health and Exercise Sciences Research Group, School of Sport,

University of Stirling, Stirling, UK longus muscles using a 2-deoxyglucose uptake technique with a physiological insulin concentration of $360 \mathrm{pmol} / 1$ $(60 \mu \mathrm{U} / \mathrm{ml})$.

Results Sirt1 mRNA and SIRT1 protein levels were increased by approximately 100- and 150-fold, respectively, in skeletal muscle of mice with SIRT1 overexpression compared with wild-type mice. Despite this large-scale overexpression of SIRT1, body composition, whole-body energy expenditure, substrate oxidation and voluntary activity were comparable between genotypes. Similarly, skeletal muscle basal and insulin-stimulated glucose uptake were unaltered with SIRT1 overexpression. Finally, while 20 days of energy restriction enhanced insulin-stimulated glucose uptake in skeletal muscles of wild-type mice, no additional effect of SIRT1 overexpression was observed.

Conclusions/interpretation These results demonstrate that upregulation of SIRT1 activity in skeletal muscle does not affect whole-body energy expenditure or enhance skeletal muscle insulin sensitivity in young mice on a standard diet with free access to chow or in young mice on energyrestricted diets.

Keywords 2-Deoxyglucose uptake - Energy restriction · Insulin sensitivity $\cdot$ SIRT1 $\cdot$ Skeletal muscle
Abbreviations
Ac-p53 Acetylated p53
AMPK AMP-activated protein kinase
CLAMS Comprehensive Lab Animal Monitoring System
Cre Cre recombinase
2DOGU 2-Deoxyglucose uptake
EDL Extensor digitorum longus
EnR Energy intake restriction
FLX $^{\text {STOP }}$ Flanking a transcriptional stop element
GA Gastrocnemius 


$\begin{array}{ll}\text { GAPDH } & \text { Glyceraldehyde-3-phosphate dehydrogenase } \\ \text { GSK } & \text { Glycogen synthase kinase } \\ \text { IR } & \text { Insulin receptor } \\ \text { MCK } & \text { Muscle creatine kinase } \\ \text { mOX } & \text { Muscle-specific SIRT1 overexpressing } \\ \text { MRI } & \text { Magnetic resonance imaging } \\ \text { p-Akt } & \text { Phosphorylated Akt } \\ \text { p-GSK } & \text { Phosphorylated GSK } \\ \text { p-IR } & \text { Phosphorylated IR } \\ \text { RER } & \text { Respiratory exchange ratio } \\ \text { SIRT } & \text { Sirtuin } \\ \text { SOL } & \text { Soleus } \\ \text { StD } & \text { Standard chow diet } \\ \dot{V} \mathrm{CO}_{2} & \text { Volume of carbon dioxide expired per unit time } \\ \dot{V} \mathrm{O}_{2} & \text { Volume of oxygen consumption } \\ \mathrm{WT} & \text { Wild-type }\end{array}$

\section{Introduction}

Skeletal muscle insulin resistance is a primary metabolic defect underlying the development of type 2 diabetes [1]. Recently, the mammalian orthologue of Sir2, sirtuin (SIRT)1, an NAD ${ }^{+}$-dependent protein deacetylase, was proposed to be a key signalling node linking alterations in energy flux to insulin action [2,3]. Mice with whole-body overexpression of SIRT1 demonstrate a metabolic phenotype similar to that seen with energy intake restriction (EnR) $[4,5]$, while pharmacological activation of SIRT1 improves skeletal muscle insulin sensitivity in rodent models of insulin resistance $[6,7]$. Although the therapeutic potential of SIRT1 activation for the treatment of skeletal muscle insulin resistance is well appreciated, studies supporting this notion have primarily been conducted in vitro or have used pharmacological activators of SIRT1 [6-9]. Given that SIRT1 is highly abundant in other key metabolic tissues (e.g. brain, adipose tissue, liver), it is possible that improvements in muscle insulin sensitivity following pharmacological treatment occur secondarily to activation of SIRT1 in these other tissues.

In L6 myotubes and primary muscle myotubes, SIRT1 overexpression has been shown to enhance insulinstimulated activation of Akt, while SIRT1 knockdown has the opposite effect [8]. However, the functional effect of these changes in Akt signalling on glucose uptake was not determined [8]. In contrast, in C2C12 myotubes, SIRT1 overexpression did not enhance insulin-stimulated activation of Akt or glucose uptake [9]. Similarly, in a gain of function mouse model that included modest overexpression of SIRT1 in skeletal muscle, glucose disposal during a hyperinsulinaemic-euglycaemic clamp was not enhanced [4]. We have also recently demonstrated in skeletal muscle that knockout of SIRT1 deacetylase activity did not impair in vivo or ex vivo insulin-stimulated glucose uptake [10]. It is clear from these studies that the precise role of SIRT1 in the regulation of skeletal muscle insulin sensitivity remains controversial. To address this, we generated mice with muscle-specific overexpression of SIRT1 (herein referred to as $\mathrm{mOX}$ mice) to determine whether increased SIRT1 activity in skeletal muscle enhances muscle insulin sensitivity. In addition, since EnR robustly enhances skeletal muscle insulin action [10-12] and increases SIRT1 activity [10], we sought to determine whether SIRT1 overexpression in conjunction with EnR would result in an additional enhancement of muscle insulin sensitivity.

\section{Methods}

Animals All studies were conducted in male mice on a C57BL/6 background. To generate $\mathrm{mOX}$ mice, mice harbouring loxP sites flanking a transcriptional stop element (FLX ${ }^{\text {STOP }}$ ) upstream of the Sirt1 gene [13] (kindly provided by D. A. Sinclair, Harvard Medical School, Boston, MA, USA) were crossed with mice expressing Cre recombinase (Cre) under the control of the muscle creatine kinase (MCK) promoter (Cre-MCK). After Cre-mediated recombination, the STOP element is removed and Sirt1 gene expression is driven by a constitutive promoter (CAGGS) that lies immediately upstream of Sirt1 [13]. The FLX ${ }^{\text {STOP }}$ mice that lack Cre-MCK are referred to herein as wild-type (WT) and were used as controls for all studies. Ex vivo measurements of basal and insulin-stimulated glucose uptake and in vivo supra-physiological insulin stimulation were performed from 13:00 to $15: 00$ hours in anaesthetised $(150 \mathrm{mg} / \mathrm{kg}$ Beuthanasia-D Special; Schering-Plough Animal Health, Union, NJ, USA) mice. For these experiments, mice with free access to a standard diet were fasted for 4 to $6 \mathrm{~h}$, while EnR mice received their food at 17:00 hours on the previous day. Mice were housed on a $12 \mathrm{~h}$ light/12 h dark cycle. All experiments were approved by and conducted in accordance with the Animal Care Program at the University of California, San Diego.

EnR diet The EnR studies were performed as previously described [10]. Briefly, at 9 weeks of age, food intake of mice with free access to a standard chow diet (catalogue number 5001; LabDiet, Brentwood, MO, USA) (StD) was measured daily at 17:00 hours for 7 days. At 10 weeks of age, mice either continued StD intake or were switched to an EnR ( $60 \%$ of StD) diet for 20 days. Food was provided daily to EnR mice between 16:00 and 17:00 hours.

Isolated muscle 2-deoxyglucose uptake Ex vivo muscle insulin sensitivity was measured by the 2-deoxyglucose uptake (2DOGU) technique in isolated soleus (SOL) and 
extensor digitorum longus (EDL) muscles as described previously [10]. The insulin concentration for insulin-treated muscles was 360 pmol/1 (60 $\mu \mathrm{U} / \mathrm{ml}$ ) (Humulin R; Eli Lilly, Indianapolis, IN, USA). Frozen SOL and EDL muscles were weighed to $0.1 \mathrm{mg}$, homogenised and 2DOGU was calculated as previously described [10].

In vivo supra-physiological insulin stimulation In anaesthetised StD-fed mice, one gastrocnemius (GA) was dissected and mice were intravenously injected with $6,000 \mathrm{pmol} / \mathrm{kg}(1 \mathrm{U} / \mathrm{kg}$ ) insulin (Humulin R; Eli Lilly) diluted in a sterile $0.9 \% \mathrm{wt} / \mathrm{vol}$. saline solution containing $1 \% \mathrm{wt} / \mathrm{vol}$. BSA. At $5 \mathrm{~min}$ after the insulin injection, the contralateral GA was dissected. Immediately after dissection, the GA was rinsed in sterile saline, blotted dry and frozen in liquid nitrogen. Samples were homogenised for SDS-PAGE as previously described [10].

Nuclear isolation Nuclear fractions were isolated from $50 \mathrm{mg}$ GA muscle using a commercially available kit (NEPER, catalogue number 78835; Thermo Fisher Scientific, Rockford, IL, USA) as per the manufacturer's instructions. The total protein concentration was determined by the bicinchoninic acid assay (Thermo Fisher Scientific).

$S D S$-PAGE SDS-PAGE was performed using standard methods, as previously described [10]. Briefly, $20 \mu \mathrm{g}$ protein was boiled in 1X SDS buffer and loaded onto 3 to $8 \%$ TRIS-acetate gels. The following primary antibodies from Cell Signaling Technology (Danvers, MA, USA) were used for immunoblotting: phosphorylated Akt (p-Akt) ${ }^{\text {Ser473 }}$, catalogue number 9271; p-Akt ${ }^{\text {Thr308 }}$, catalogue number 9275 ; Akt, catalogue number 9272; p53, catalogue number 2524; glycogen synthase kinase (GSK)- $3 \alpha / \beta$, catalogue number 5676; phosphorylated GSK (p-GSK)-3 $\alpha / \beta^{\text {Ser21/9 }}$, catalogue

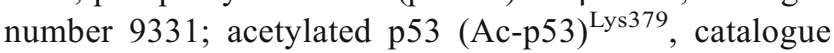
number 2570; insulin receptor (IR) $\beta$, catalogue number 3025; phosphorylated IR (p-IR) $\beta^{\text {Tyr1150/1151 }}$, catalogue no. 3024. Other primary antibodies used were: histone H2B, catalogue number ab9408 (AbCam, Cambridge, MA, USA) and glyceraldehyde-3-phosphate dehydrogenase (GAPDH), catalogue number 10R-G109a (Fitzgerald Industries, Acton, MA, USA).

Real-time RT-PCR RNA was extracted from EDL, GA, plantaris and SOL muscles, and from liver and adipose tissue using the phenol/chloroform method. cDNA was synthesised from $1 \mu \mathrm{g}$ of RNA using a kit (i-Script cDNA Synthesis, catalogue number 170-8891; Bio-Rad Laboratories, Hercules, CA, USA). Real-time RT-PCR was performed using a Mastercycler ep realplex (Eppendorf, Hamburg, Germany), SYBR Green Taq Polymerase (catalogue number 17-8880; Bio-Rad) and custom-designed primers. Target gene expression for each sample was calculated relative to Gapdh. Primer sequences were as follows: Gapdh 5'-ATTCAACGGCACAGTCAAG-3' and 3'-TCCACGACATACTCAGCAC-5'; Sirt 1 5'-GGCT ACCGAGACAACCTCCTG-3' and 3'-AGTCCAGTCAC TAGAGCTGGC-5'.

Blood glucose and plasma insulin Fasting blood glucose was measured in conscious mice (Ascensia Contour; Bayer Healthcare, Mishawaka, IN, USA) before anaesthesia for 2DOGU. Plasma insulin concentrations were measured using a commercially available kit (Mouse Ultrasensitive Insulin ELISA, catalogue number 80-INSMSU-E01; Alpco Diagnostics, Salem, NH, USA) according to the manufacturer's instructions. For this, blood was collected in EDTA from the inferior vena cava of anaesthetised mice and centrifuged $\left(5,000 \mathrm{~g}, 5 \mathrm{~min}, 4^{\circ} \mathrm{C}\right)$, and the supernatant fraction was frozen $\left(-80^{\circ} \mathrm{C}\right)$ for subsequent measurement.

Measurement of AMP-activated protein kinase activity GA muscle powdered under liquid nitrogen was lysed by shaking on an orbital shaker $\left(1,500 \mathrm{~g}, 4^{\circ} \mathrm{C}\right)$ in AMP-activated protein kinase (AMPK) lysis buffer $(50 \mathrm{mmol} / \mathrm{l}$ Tris- $\mathrm{HCl}$, $\mathrm{pH} 7.5,150 \mathrm{mmol} / \mathrm{l} \mathrm{NaCl}, 50 \mathrm{mmol} / \mathrm{l} \mathrm{NaF}, 5 \mathrm{mmol} / \mathrm{l}$ sodium pyrophosphate, $1 \mathrm{mmol} / 1 \mathrm{EDTA}, 1 \mathrm{mmol} / 1 \mathrm{EGTA}, 1 \mathrm{mmol} / 1$ dithiothreitol, $0.1 \mathrm{mmol} / 1$ benzamidine) with $0.1 \mathrm{mmol} / 1$ phenylmethanesulfonyl fluoride, $5 \mu \mathrm{g} / \mathrm{ml}$ soybean trypsin inhibitor and $1 \%$ vol./vol. Triton X-100. Lysates were cleared by centrifugation for $15 \mathrm{~min}$ at $16,200 \mathrm{~g}$ and $4^{\circ} \mathrm{C}$. Protein content was determined (bicinchoninic acid assay; Fisher Scientific, Loughborough, UK). AMPK activity was determined as previously described [14]. Briefly, AMPK $\alpha 1$ and $\alpha 2$ antibodies (a kind gift from G. Hardie, University of Dundee, Dundee, Scotland, UK) were used to independently immunoprecipitate $\alpha 1$ and $\alpha 2$ complexes. AMPK activity was calculated as the difference in counts between AMARA (AMPK substrate: AMARAASAAALARRR)-containing and AMARA-negative samples as pmol ATP incorporated per min per mg protein (or $\mathrm{mU} / \mathrm{mg}$ ).

Energy expenditure and body composition The volume of oxygen consumption $\left(\dot{V} \mathrm{O}_{2}\right)$, the volume of carbon dioxide expired per unit time $\left(\dot{V} \mathrm{CO}_{2}\right)$, the respiratory exchange ratio (RER), physical activity (total movement [x-total], ambulatory activity $[\mathrm{x}-\mathrm{amb}]$, rearing activity [z-total]) and food intake were assessed using the Comprehensive Lab Animals Monitoring System (CLAMS) (Columbus Instruments, Columbus, $\mathrm{OH}, \mathrm{USA}$ ). Measurements were made for three consecutive days and values were averaged from the light and dark phases recorded on days 2 and 3. Fat oxidation $(\mathrm{kJ} / \mathrm{h})$ was calculated from values for RER and heat $(\mathrm{kJ} / \mathrm{h})$, as previously described [15]. Body composition 
was measured by magnetic resonance imaging (MRI) (EchoMRI, Houston, TX, USA).

Statistics Statistical analyses were performed using SigmaPlot 11.2 (Systat Software, Chicago, IL, USA). Data were analysed by two-way ANOVA (with repeated measures when necessary) for main effects of diet and genotype, followed by Tukey's post-hoc analysis, with significant differences at $p<0.05$. Since for 2DOGU and insulin signalling data, there was an effect of insulin vs basal for all groups, a two-way ANOVA was performed within basal and within insulin samples. All data are expressed as mean $\pm \mathrm{SEM}$.

\section{Results}

Muscle-specific SIRT1 overexpression Sirt1 mRNA levels were approximately 100-fold higher in skeletal muscle from mOX vs WT mice, but were comparable in adipose tissue and liver (Fig. 1a). SIRT1 protein levels were approximately 150 times higher than WT in soleus and EDL muscles from mOX mice (Fig. 1b). To determine whether this large-scale overexpression of SIRT1 resulted in a functional increase in SIRT1 activity, we measured Ac-p53 ${ }^{\text {Lys379 }}$, a known target of SIRT1 [16], in nuclear lysates from GA muscle. In line with the increased gene and protein expression of SIRT1 in mOX mice, nuclear Ac-p53 ${ }^{\text {Lys } 379}$ :total p53 was significantly reduced by approximately $35 \%$ in $\mathrm{mOX}$ vs WT mice (Fig. 1c). Total p53 abundance in the nucleus was approximately $30 \%$ higher in mOX vs WT mice (WT $1.00 \pm 0.01$, $\operatorname{mOX} 1.31 \pm 0.11, p<0.05, n=4$ ) (Fig. 1c). However, there was no difference in histone $\mathrm{H} 2 \mathrm{~B}$ abundance, indicating equal loading of the nuclear fraction (WT $1.00 \pm 0.03$, $\operatorname{mOX} 1.02 \pm 0.01, p>0.05, n=4$ ) (Fig. 1c).
Energy expenditure, spontaneous activity, food intake and supra-physiological insulin signalling are not altered in $m O X$ mice While expected diurnal variations were observed, large-scale overexpression of SIRT1 in skeletal muscle did not result in alterations in $\dot{V} \mathrm{O}_{2}$, RER or fat oxidation in $\mathrm{mOX}$ mice (Fig. 2a-c). Moreover, the number of recorded beam breaks as a measure of total spontaneous activity (Fig. 2d), ambulatory activity (Light: WT $45 \pm 2$, mOX $70 \pm 16$ beam breaks; Dark: WT 254 \pm 66 , mOX $271 \pm 25$ beam breaks, $p>0.05$ ) and rearing activity (Light: WT $9 \pm 21, \operatorname{mOX} 13 \pm 5$ beam breaks; Dark: WT $97 \pm 45$, mOX $132 \pm 32$ beam breaks, $p>0.05$ ) did not differ between genotypes. Food intake was similar in WT and mOX mice (Light: WT $1.34 \pm 0.16 \mathrm{~g}, \operatorname{mOX} 1.27 \pm 0.12 \mathrm{~g}$; Dark: WT $3.01 \pm 0.24 \mathrm{~g}, \operatorname{mOX} 3.37 \pm 0.11 \mathrm{~g}, p>0.05)$. In addition, supra-physiological, in vivo insulin stimulation for $5 \mathrm{~min}$ resulted in robust increases in phosphorylation of $\operatorname{IR} \beta$ $\left(\mathrm{p}-\mathrm{IR} \beta^{\text {Tyr1 150/1151 }}\right)$, Akt (p-Akt ${ }^{\mathrm{Ser} 473}$ and $\left.\mathrm{p}-\mathrm{Akt}^{\mathrm{Thr} 308}\right)$ and GSK3 $\beta$ (p-GSK $3 \beta^{\text {Ser9 }}$ ) above basal in GA muscle, but there were no differences in these variables between genotypes (Fig. 2e, f).

Body composition, fasting blood variables and skeletal muscle AMPK activity are not altered in $\mathrm{mOX}$ mice $\mathrm{In} \mathrm{StD}-\mathrm{fed}$ mice, body weight, lean mass, fat mass and per cent body fat did not differ between WT and mOX mice. With EnR, these variables decreased similarly in both genotypes (Fig. 3a, b). No effect of genotype on fasting glucose concentrations was observed in StD-fed mice, while EnR decreased fasting glucose levels to similar degrees in WT and mOX mice (Fig. 3c). No significant effects of diet or genotype on plasma insulin concentrations were observed (WT-StD $69 \pm 7$, WTEnR 42 \pm 6, mOX-StD $42 \pm 4$, mOX-EnR $46 \pm 11 \mathrm{pmol} / 1$, $p>0.05, n=6-10)$. We also found no effect of diet or genotype on AMPK $\alpha 1$ or $\alpha 2$ activity (Fig. $3 \mathrm{~d}$ ).

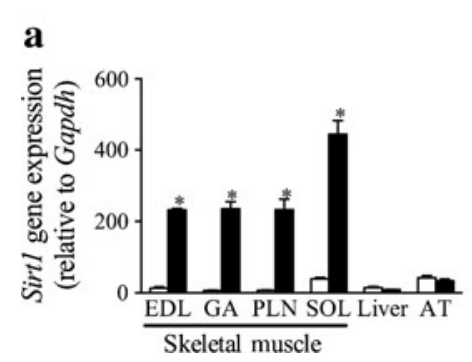

Fig. 1 mOX mice display increased SIRT1 activity in skeletal muscle. (a) mRNA levels of Sirt1 in SOL, EDL, GA, plantaris (PLN), liver and adipose tissue (AT) were measured relative to Gapdh by real-time RTPCR in WT (white bars) and mOX mice (black bars) $(n=3)$. (b) SIRT1 protein levels were measured in soleus and EDL muscles by immunoblot and quantified relative to GAPDH $(n=6-8)$. Data are presented as
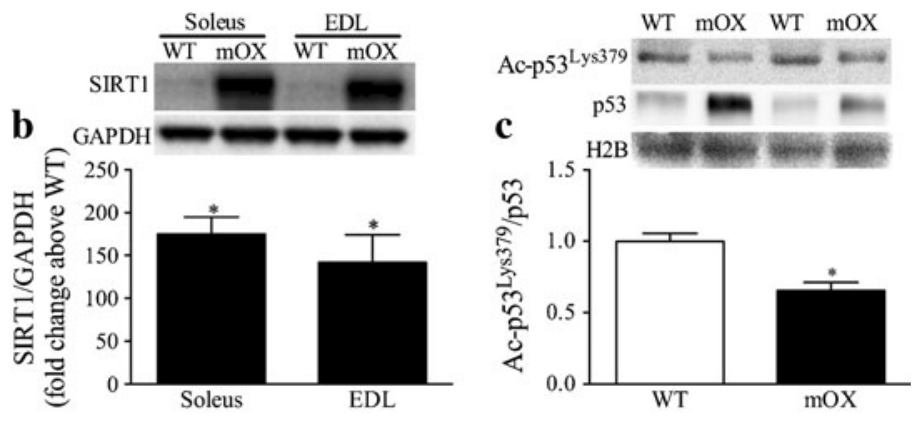

fold change of mOX above WT. (c) Ac-p53 ${ }^{\text {Lys379 }}$, total p53 and histone H2B were analysed by SDS-PAGE in nuclear fractions from GA muscle $(n=4)$. Quantification is of Ac-p53 $3^{\text {Lys379 }}$ corrected for total p53 abundance. Values are mean \pm SEM; $* p<0.05$ for effect of genotype 

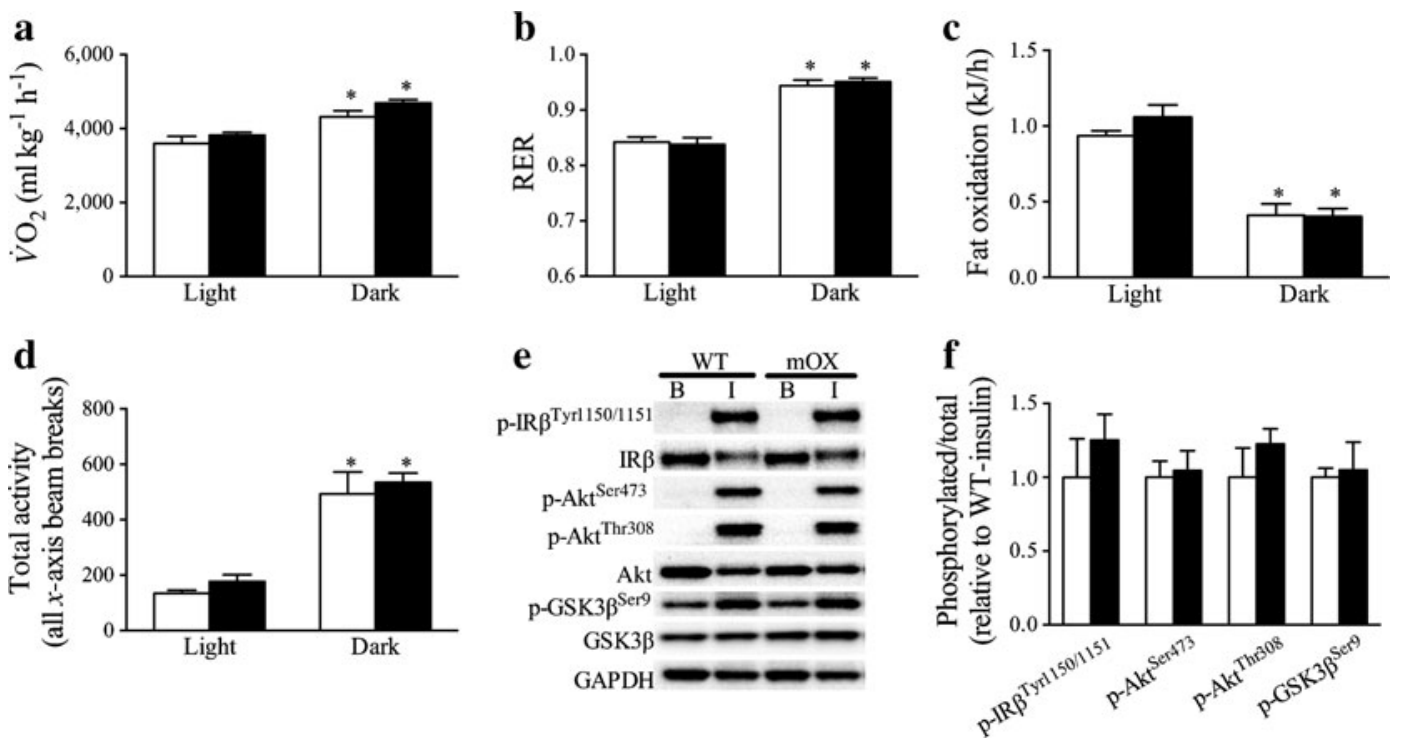

Fig. 2 Overexpression of SIRT1 in skeletal muscle does not alter energy expenditure, spontaneous activity or in vivo insulin signalling. (a-d) Measurements were made using the CLAMS system over 3 consecutive days with averages for the light and dark cycles on days 2 and 3 displayed for WT (white bars) and $\mathrm{mOX}$ (black bars) mice $(n=$ 6-8). (a) $\dot{V} \mathrm{O}_{2}$ and (b) RER were measured by indirect calorimetry. (c) Fat oxidation was calculated from indirect calorimetry data as described by others [15]. (d) Total (x-total) activity was measured as

Skeletal muscle insulin sensitivity and signalling are not enhanced in $\mathrm{mOX}$ mice To determine the effects of muscle-specific SIRT1 overexpression on insulin-stimulated
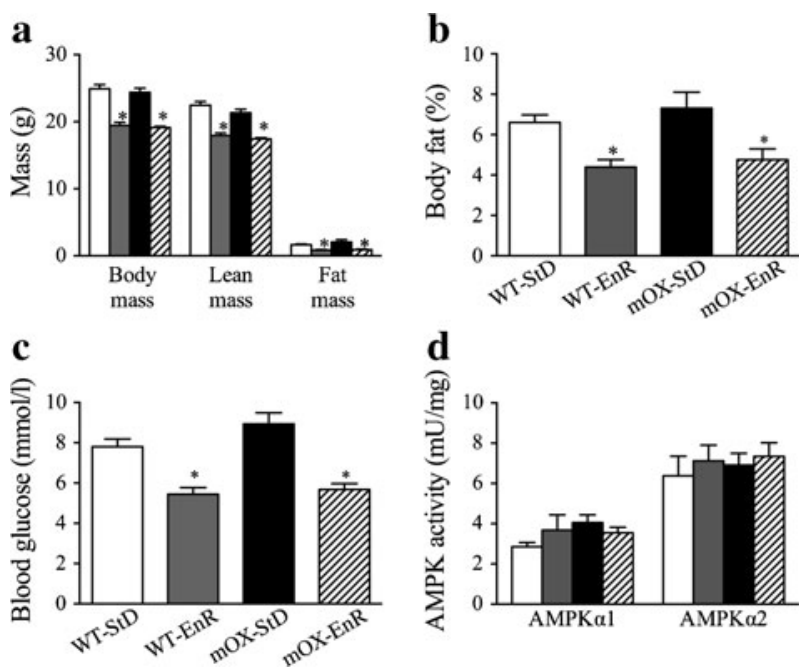

Fig. 3 Metabolic profiles and skeletal muscle AMPK activity in WT and mOX mice. Beginning at 10 weeks of age, WT and mOX mice were fed a StD or EnR diet for 20 days. White bars, WT-StD; grey bars, WT-EnR; black bars, mOX-StD; hatched bars, mOX-EnR. (a) Body mass was measured to $0.1 \mathrm{~g}$, and lean and fat mass were determined by MRI $(n=10-19)$. (b) Per cent body fat was calculated from fat and body mass measurements $(n=9-19)$. (c) Basal glucose levels $(n=10-19)$ in fasted mice. (d) AMPK $\alpha 1$ and $\alpha 2$ complexes were immunoprecipitated and their activities quantified $(n=6)$ in GA muscle. Values are mean $\pm \mathrm{SEM} ;{ }^{*} p<0.05$ for effect of diet all beam breaks on the horizontal axis. (e) Representative immunoblot images and (f) quantified data for phosphorylated and total IR $\beta$, Akt and GSK $3 \beta$ in basal GA muscle and GA muscle collected 5 min after supra-physiological $(6,000 \mathrm{pmol} / \mathrm{kg})$ insulin stimulation (B, basal; I, insulin). Phosphorylated levels were quantified relative to total levels $(n=4)$; GAPDH was used as a loading control. Data (f) are presented relative to WT-insulin. Values are mean \pm SEM; $* p<0.05$ for effect of time (a-d)

glucose uptake, we measured basal and insulin-stimulated glucose uptake ex vivo in isolated SOL and EDL muscles. We observed no effects of genotype or diet on basal glucose uptake (WT-StD $0.45 \pm 0.03$, WT-EnR $0.39 \pm 0.04$, mOXStD $0.36 \pm 0.06$, mOX-EnR $0.34 \pm 0.06 \mu \mathrm{mol}[20 \mathrm{~min}]^{-1}$ [g muscle $^{-1}, p>0.05$ ). Insulin-stimulated 2DOGU (insulin 2DOGU-basal 2DOGU) in SOL and EDL muscles did not differ between WT and mOX mice on an StD diet (Fig. 4a). Moreover, while EnR enhanced insulin-stimulated 2DOGU in SOL and EDL muscles as compared with StD in both genotypes, no additional effect on insulin sensitivity was seen in mOX mice (Fig. 4a). Complementing the 2DOGU findings, insulin-stimulated $\mathrm{p}-\mathrm{Akt}^{\mathrm{Ser} 473}$ and $\mathrm{p}-\mathrm{Akt}^{\mathrm{Thr} 308}$ in SOL muscle were enhanced by EnR above StD in WT and mOX mice (Fig. 4b, c). Downstream of Akt signalling, insulin-stimulated $\mathrm{p}$-GSK-3 $\beta^{\text {Ser9 }}$ was enhanced during EnR vs StD in WT mice and showed a trend $(p=0.054)$ to increase in mOX-EnR mice (Fig. 4b, c). Notably, in insulintreated muscles from EnR mice, p-Akt ${ }^{\text {Thr308 }}$ and p-GSK$3 \beta^{\text {Ser9 }}$ were significantly lower, and $\mathrm{p}-\mathrm{Akt}^{\mathrm{Ser} 473}$ showed a trend to be lower $(p=0.065)$ in mOX vs WT mice (Fig. $4 \mathrm{~b}, \mathrm{c})$. In StD-fed mice, there were no differences in $\mathrm{p}-\mathrm{Akt}^{\mathrm{Ser} 473}$, p-Akt ${ }^{\text {Thr308 }}$ or p-GSK-3 $\beta^{\text {Ser9 }}$ between genotypes (Fig. $4 \mathrm{~b}$, c). Moreover, no effects of EnR or genotype were observed on total Akt or GSK3 $\beta$ abundance (Fig. 4b), or on basal: (1) $\mathrm{p}^{-\mathrm{Akt}^{\mathrm{Ser}} 473}$ (WT-StD $0.13 \pm 0.03$, WT-EnR $0.07 \pm 0.01$, mOX-StD $0.07 \pm 0.02, \mathrm{mOX}-\mathrm{EnR} 0.08 \pm 0.03$, relative to WT-StD insulin); (2) p-Akt ${ }^{\text {Thr308 }}$ (WT-StD $0.08 \pm 0.02$, 


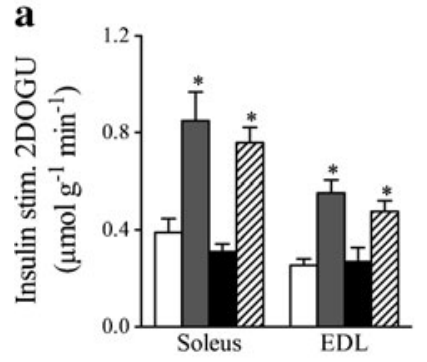

b

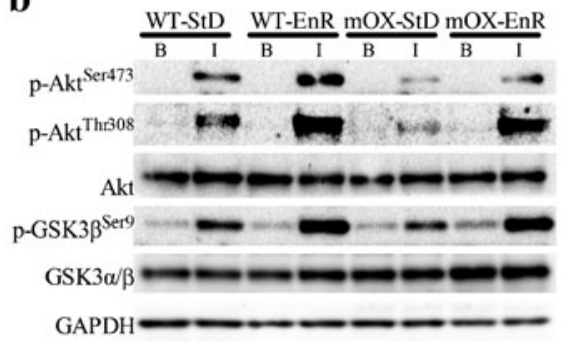

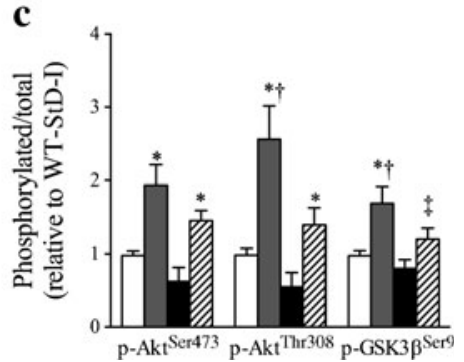

Fig. 4 Insulin sensitivity and signalling are not enhanced in mOX mice. Beginning at 10 weeks of age, WT and mOX mice were fed a StD or EnR diet for 20 days. White bars, WT-StD; grey bars, WT-EnR; black bars, mOX-StD; hatched bars, mOX-EnR. (a) Insulin-stimulated 2DOGU (insulin 2DOGU [360 pmol/1] - basal 2DOGU) in soleus and EDL muscles $(n=7-11)$ was assessed after the 20 day diet period. (b) Insulin-stimulated Akt and GSK $3 \beta$ activation were measured in soleus

WT-EnR $0.05 \pm 0.01$, mOX-StD $0.08 \pm 0.02$, mOX-EnR $0.10 \pm 0.03$, relative to WT-StD insulin); or (3) p-GSK$3 \beta^{\text {Ser9 }}$ (WT-StD $0.37 \pm 0.03$, WT-EnR $0.33 \pm 0.04$, mOX-StD $0.37 \pm 0.05$, mOX-EnR $0.31 \pm 0.05$, relative to WT-StD insulin).

\section{Discussion}

SIRT1 has been proposed to be an important regulator of insulin sensitivity in key insulin target tissues, including skeletal muscle [2,3]. To date, however, the precise role of SIRT1 in the regulation of skeletal muscle insulin sensitivity has not been fully elucidated. To address this, we generated mice with muscle-specific overexpression of SIRT1. Our results demonstrate that large-scale overexpression of SIRT1 in skeletal muscle does not enhance muscle insulin sensitivity or alter energy expenditure in young, lean mice. In addition, SIRT1 overexpression in EnR mice did not further enhance skeletal muscle insulin action. Taken together, these results suggest that augmenting SIRT1 activity in skeletal muscle does not increase skeletal muscle insulin sensitivity or whole-body energy expenditure in young, lean mice.

The therapeutic potential of SIRT1 activation for the treatment of muscle insulin resistance has been proposed for some years [2, 3]. Accordingly, treatment of obese rodents with SIRT1-specific activators has been demonstrated to improve in vivo skeletal muscle insulin action in response to a supra-physiological insulin infusion $[6,7]$. An important limitation of pharmacological studies is that activation of SIRT1 occurs in other metabolic tissues, including adipose tissue, liver and brain. Thus, it is not possible to determine whether improved muscle insulin sensitivity is due to direct effects in muscle or whether it manifests secondarily to SIRT1 activation in these other tissues. Indeed, SIRT1 activation inhibits inflammatory pathways in macrophages and reduces adipose tissue inflammation in muscle lysate by immunoblotting for p-Akt ${ }^{\mathrm{Ser} 473}(n=5-9)$, p-Akt ${ }^{\mathrm{Thr} 308}$ $(n=7-11)$ and p-GSK-3 $\beta^{\operatorname{Ser} 9}(n=6-9)$. B, basal; I, insulin-stimulated. (c) Data are displayed as p-Akt ${ }^{\mathrm{Ser} 473} /$ total Akt, p-Akt ${ }^{\text {Thr308 }} /$ total Akt and $\mathrm{p}-\mathrm{GSK}-3 \beta^{\mathrm{Ser} 9} /$ total GSK $3 \beta$, respectively. GAPDH was used as a loading control. All samples were normalised to WT-StD-insulin. Values are mean $\pm \mathrm{SEM} ;{ }^{*} p<0.05$ for effect of diet; ${ }^{\dagger} p<0.05$ for effect of genotype; ${ }^{\star} p=0.054$ for effect of diet

obese rodents [17, 18], which can have beneficial effects on peripheral insulin sensitivity, as reviewed by others [19]. Few studies have directly investigated the role of SIRT1 in skeletal muscle insulin action. Frojdo and colleagues demonstrated in primary muscle and L6 myotubes that SIRT1 overexpression enhances, while SIRT1 knockdown impairs insulin-stimulated activation of Akt [8]. Insulin-stimulated glucose uptake was not determined in this study, so it is not known whether these changes in Akt signalling resulted in functional alterations in insulin sensitivity. Additionally, the authors used supra-physiological (10,000-100,000 pmol/l) insulin concentrations, which may not accurately represent alterations in insulin sensitivity under physiological conditions. Our results clearly demonstrate that SIRT 1 overexpression does not enhance skeletal muscle insulin action in response to a physiological insulin concentration. Supporting our findings, SIRT1 overexpression in C2C12 myotubes did not enhance insulin-stimulated glucose uptake or Akt signalling at a supra-physiological (100 nmol/l) insulin concentration [9]. Similarly, we found no difference in in vivo insulin signalling in response to a supraphysiological insulin dose. In addition, knockout of SIRT1 deacetylase activity in skeletal muscle did not impair in vivo or ex vivo insulin-stimulated glucose uptake, although SIRT1 is required for EnR to enhance muscle insulin action [10]. Taken together, while cell-based studies potentially support a role for SIRT1 in the regulation of muscle insulin action, studies in bona fide skeletal muscle question a possible role for SIRT1 in the regulation of insulin action in this tissue under physiological conditions.

In this investigation, we studied lean, young mice; however, it is possible that any beneficial effects of muscle SIRT1 overexpression may only manifest in an insulinresistant model. Indeed, when $\mathrm{C} 2 \mathrm{C} 12$ myotubes were stimulated with 100,000 pmol/1 insulin, SIRT1 overexpression prevented fatty-acid-induced insulin resistance [9]. In contrast to these studies in $\mathrm{C} 2 \mathrm{C} 12$ myotubes, Banks et al [4] 
found in a whole-body SIRT1 gain of function model that skeletal muscle insulin sensitivity (as measured by a hyperinsulinaemic-euglycaemic clamp) was not improved in 11-month-old mice or in a high-fat diet model of insulin resistance. These findings provide additional support for the contention that SIRT1 does not directly regulate skeletal muscle insulin action, even in models of insulin resistance, though studies in a muscle-specific model, such as the mOX mouse, will help to add weight to this line of argument. It is important to acknowledge that germline overexpression of SIRT1 occurs in our model, as well as large-scale (i.e. approximately 150 -fold) SIRT1 overexpression. Thus, it is possible that chronic overexpression of SIRT1 or such large-scale overexpression may preempt changes that are beneficial to insulin action. Notably, however, in the aforementioned study by Banks et al [4], muscle insulin action was not affected by two- to threefold SIRT1 overexpression from germline.

EnR enhances insulin-stimulated glucose uptake due in part to greater activation of insulin signalling at the level of phosphatidylinositol 3 kinase $[10,12]$ or Akt $[10,12,20]$. As expected, in WT mice we observed beneficial effects of EnR on insulin-stimulated glucose uptake and on activation of insulin signalling at Akt and its downstream target GSK $3 \beta$. Since we have previously demonstrated that skeletal muscle SIRT1 deacetylase activity is required for the effects of EnR on muscle insulin sensitivity to occur [10], and mice with whole-body overexpression of SIRT1 demonstrate a metabolic phenotype similar to that seen with EnR $[4,5]$, we hypothesised that an increase in SIRT1 activity in combination with EnR would have an additional effect on muscle insulin action. However, we observed no additional effect on muscle insulin-stimulated glucose uptake during EnR in mOX mice in response to a physiological insulin concentration. We interpret these data to suggest that endogenous levels of SIRT1 are sufficient to maximise the ability of EnR to enhance muscle insulin action. Interestingly, in muscle from EnR mice, but not in that from StD mice, insulin-stimulated activation of $\mathrm{p}-\mathrm{Akt}^{\mathrm{Thr} 308}$ and $\mathrm{p}$ GSK $3 \alpha / \beta$ was reduced in mOX compared with WT mice. While the reasons for this are not readily apparent, measurements of insulin signalling were made in muscles that were stimulated with insulin for $50 \mathrm{~min}$, and if $\mathrm{mOX}$ mice display faster insulin signalling kinetics, these signals may be turned off faster in mOX mice with EnR. Indeed, we found no differences in the activation of insulin signalling at the level of IR, Akt or GSK3 $\beta$ between WT and $\mathrm{mOX}$ mice at $5 \mathrm{~min}$ after a supraphysiological insulin dose.

Since SIRT1 is thought to be involved in the regulation of mitochondrial biogenesis in muscle [21, 22], it is possible that SIRT1 overexpression in skeletal muscle may affect whole-body energy expenditure and/or substrate utilisation. In line with this, energy expenditure is slightly elevated in mice with moderate whole-body (including skeletal muscle) overexpression of SIRT1 [23]. In contrast, mice with SIRT1 gain of function that resulted in modest SIRT1 overexpression in skeletal muscle demonstrate decreased oxygen consumption [4]. Intriguingly, whole-body SIRT1null mice display increased metabolic rates and a decrease in RER [24]. The reasons for such contrasting results are not readily apparent, but may be due to the different strains of mice used or to the way in which SIRT1 was manipulated. In any case, the above-mentioned studies certainly suggest that SIRT1 can play a role in the regulation of whole-body energy expenditure, although they do not reveal which tissue or tissues contribute to this effect. In a mouse model of SIRT1 overexpression in which SIRT1 was not overexpressed in muscle or liver, increased oxygen consumption was observed [5], suggesting that tissues other than muscle or liver may mediate the aforementioned effects of SIRT1 on energy expenditure. In further support of this notion, decreased energy expenditure was observed in a recent study using brain-specific knockdown of SIRT1 [25]. Our present results demonstrate that skeletal muscle SIRT1 overexpression does not affect whole-body energy expenditure, RER or fat oxidation. Additionally, we have found that loss of SIRT1 deacetylase activity in mouse skeletal muscle has no effect on energy expenditure (White A.T., Schenk S., unpublished observations). Thus, skeletal muscle SIRT1 does not affect energy expenditure and reported alterations in energy expenditure in models with manipulation of SIRT1 are likely to be due to adaptations in tissues other than muscle.

SIRT1 has been proposed to be an important regulator of AMPK activity through its ability to regulate liver kinase B1 [26-28], while other studies have demonstrated AMPK to be an important regulator of SIRT1 [29, 30]. AMPK has also been observed to be activated by EnR and consequently is thought to mediate the beneficial effects of EnR on insulin sensitivity [31, 32]. Here, we measured skeletal muscle AMPK ( $\alpha 1$ and $\alpha 2)$ activity to determine whether it was altered in response to SIRT1 overexpression and/or EnR. We observed no effect of SIRT1 overexpression or EnR on skeletal muscle AMPK $\alpha 1$ or $\alpha 2$ activity, which is in line with previous observations that AMPK activation is not altered in skeletal muscle during EnR [10, 12, 20, 33]. Combined with our recent findings demonstrating that knockout of SIRT1 deacetylase activity had no effect on AMPK activation [10,34], the present results suggest that SIRT1 is not a major regulator of AMPK activity in skeletal muscle.

In summary, the present study demonstrates that SIRT1 overexpression in skeletal muscle does not enhance skeletal muscle insulin signalling or insulin sensitivity, affect 
AMPK activity, or alter whole-body energy expenditure. Furthermore, while EnR robustly improves muscle insulin action, SIRT1 overexpression has no additional effect above and beyond that achieved by EnR alone. Together with other recent studies [4], these data demonstrate that overexpression of SIRT1 does not improve muscle insulin action in young mice.

Acknowledgements The authors are grateful to E.H. Buckner, B. Huang and T.R. Kim (Department of Orthopaedic Surgery, University of California, San Diego, CA, USA) for assistance with mouse studies, and to D.A. Sinclair (Harvard Medical School, Boston, MA, USA) for providing the FLX ${ }^{\text {STOP }}$ mouse. We also thank J. Shao (Department of Pediatrics, University of California, San Diego, CA, USA) for use of the MRI machine.

Funding This work was supported by grants from the National Institutes of Health (NIH), the UCSD Academic Senate and the Society for Endocrinology. D.L. Hamilton is supported by a Society for Endocrinology Early Career Grant. S. Schenk and this work were supported by grants from the NIH (R01 AG043120, R24 HD050837, P30 AR058878), the UCSD Academic Senate (RL131M) and a Pilot and Feasibility grant from the UCSD-University of California, Los Angeles [UCLA]-Cedars-Sinai/Salk Diabetes and Endocrinology Research Center (P30 DK063491). C.E. McCurdy is supported by grants from the NIH (P30 DK048520, P30 DK57516) and the Office of Research in Women's Health (K12 HD057022).

Duality of interest The authors declare that there is no duality of interest associated with this manuscript.

Contribution statement SS and ATW were responsible for the conception and design of the study, and the analysis and interpretation of data. ATW was responsible for design and drafting of the manuscript and SS revised the manuscript critically. CEM, AP, DLH and CDJ were responsible for the analysis and interpretation of data, and revised the manuscript critically. All authors gave final approval.

\section{References}

1. Weyer C, Tataranni PA, Bogardus C, Pratley RE (2001) Insulin resistance and insulin secretory dysfunction are independent predictors of worsening of glucose tolerance during each stage of type 2 diabetes development. Diabetes Care 24:89-94

2. Houtkooper RH, Canto C, Wanders RJ, Auwerx J (2010) The secret life of NAD+: an old metabolite controlling new metabolic signaling pathways. Endocr Rev 31:194-223

3. Yu J, Auwerx J (2010) Protein deacetylation by SIRT1: an emerging key post-translational modification in metabolic regulation. Pharmacol Res 62:35-41

4. Banks AS, Kon N, Knight C et al (2008) SirT1 gain of function increases energy efficiency and prevents diabetes in mice. Cell Metab 8:333-341

5. Bordone L, Cohen D, Robinson A et al (2007) SIRT1 transgenic mice show phenotypes resembling calorie restriction. Aging Cell 6:759-767

6. Feige JN, Lagouge M, Canto C et al (2008) Specific SIRT1 activation mimics low energy levels and protects against dietinduced metabolic disorders by enhancing fat oxidation. Cell Metab 8:347-358
7. Milne JC, Lambert PD, Schenk S et al (2007) Small molecule activators of SIRT1 as therapeutics for the treatment of type 2 diabetes. Nature 450:712-716

8. Frojdo S, Durand C, Molin L et al (2011) Phosphoinositide 3-kinase as a novel functional target for the regulation of the insulin signaling pathway by SIRT1. Mol Cell Endocrinol 335:166-176

9. Sun C, Zhang F, Ge X et al (2007) SIRT1 improves insulin sensitivity under insulin-resistant conditions by repressing PTP1B. Cell Metab 6:307-319

10. Schenk S, McCurdy CE, Philp A et al (2011) Sirt1 enhances skeletal muscle insulin sensitivity in mice during caloric restriction. J Clin Invest 121:4281-4288

11. Cartee GD, Dean DJ (1994) Glucose transport with brief dietary restriction: heterogenous responses in muscles. Am J Physiol 266: E946-E952

12. McCurdy CE, Cartee GD (2005) Akt2 is essential for the full effect of calorie restriction on insulin-stimulated glucose uptake in skeletal muscle. Diabetes 54:1349-1356

13. Firestein R, Blander G, Michan S et al (2008) The SIRT1 deacetylase suppresses intestinal tumorigenesis and colon cancer growth. PLoS One 3:e2020

14. McGee SL, Mustard KJ, Hardie DG, Baar K (2008) Normal hypertrophy accompanied by phosphorylation and activation of AMP-activated protein kinase alpha1 following overload in LKB1 knockout mice. J Physiol 586:1731-1741

15. Bruss MD, Khambatta CF, Ruby MA, Aggarwal I, Hellerstein MK (2010) Calorie restriction increases fatty acid synthesis and whole body fat oxidation rates. Am J Physiol Endocrinol Metab 298: E108-E116

16. Yi J, Luo J (2010) SIRT1 and p53, effect on cancer, senescence and beyond. Biochim Biophys Acta 1804:1684-1689

17. Yoshizaki T, Schenk S, Imamura T et al (2010) SIRT1 inhibits inflammatory pathways in macrophages and modulates insulin sensitivity. Am J Physiol Endocrinol Metab 298:E419-E428

18. Yoshizaki T, Milne JC, Imamura T et al (2009) SIRT1 exerts anti-inflammatory effects and improves insulin sensitivity in adipocytes. Mol Cell Biol 29:1363-1374

19. Osborn O, Olefsky JM (2012) The cellular and signaling networks linking the immune system and metabolism in disease. Nat Med 18:363-374

20. Sharma N, Arias EB, Bhat AD et al (2011) Mechanisms for increased insulin-stimulated Akt phosphorylation and glucose uptake in fast- and slow-twitch skeletal muscles of calorierestricted rats. Am J Physiol Endocrinol Metab 300:E966-E978

21. Nogueiras R, Habegger KM, Chaudhary N et al (2012) Sirtuin 1 and sirtuin 3: physiological modulators of metabolism. Physiol Rev 92:1479-1514

22. Menzies KJ, Hood DA (2012) The role of SirT1 in muscle mitochondrial turnover. Mitochondrion 12:5-13

23. Pfluger PT, Herranz D, Velasco-Miguel S, Serrano M, Tschop MH (2008) Sirt1 protects against high-fat diet-induced metabolic damage. Proc Natl Acad Sci USA 105:9793-9798

24. Boily G, Seifert EL, Bevilacqua L et al (2008) SirT1 regulates energy metabolism and response to caloric restriction in mice. PLoS One 3:e1759

25. Ramadori G, Fujikawa T, Fukuda M et al (2010) SIRT1 deacetylase in POMC neurons is required for homeostatic defenses against diet-induced obesity. Cell Metab 12:78-87

26. Lan F, Cacicedo JM, Ruderman N, Ido Y (2008) SIRT1 modulation of the acetylation status, cytosolic localization, and activity of LKB1. Possible role in AMP-activated protein kinase activation. J Biol Chem 283:27628-27635

27. Suchankova G, Nelson LE, Gerhart-Hines $Z$ et al (2009) Concurrent regulation of AMP-activated protein kinase and SIRT1 in mammalian cells. Biochem Biophys Res Commun 378:836-841 
28. Hou X, Xu S, Maitland-Toolan KA et al (2008) SIRT1 regulates hepatocyte lipid metabolism through activating AMP-activated protein kinase. J Biol Chem 283:20015-20026

29. Canto C, Gerhart-Hines Z, Feige JN et al (2009) AMPK regulates energy expenditure by modulating NAD + metabolism and SIRT1 activity. Nature 458:1056-1060

30. Canto C, Jiang LQ, Deshmukh AS et al (2010) Interdependence of AMPK and SIRT1 for metabolic adaptation to fasting and exercise in skeletal muscle. Cell Metab 11:213-219

31. Palacios OM, Carmona JJ, Michan S et al (2009) Diet and exercise signals regulate SIRT3 and activate AMPK and PGC-1alpha in skeletal muscle. Aging 1:771-783
32. Wang P, Zhang RY, Song J et al (2012) Loss of AMP-activated protein kinase-alpha2 impairs the insulin-sensitizing effect of calorie restriction in skeletal muscle. Diabetes 61:1051-1061

33. Gonzalez AA, Kumar R, Mulligan JD, Davis AJ, Weindruch R, Saupe KW (2004) Metabolic adaptations to fasting and chronic caloric restriction in heart, muscle, and liver do not include changes in AMPK activity. Am J Physiol Endocrinol Metab 287:E1032-E1037

34. Philp A, Chen A, Lan D et al (2011) Sirtuin 1 (SIRT1) deacetylase activity is not required for mitochondrial biogenesis or peroxisome proliferator-activated receptor-gamma coactivator-1alpha (PGC1alpha) deacetylation following endurance exercise. J Biol Chem 286:30561-30570 